\title{
autodE: Automated Calculation of Reaction Energy Profiles - Application to Organic and Organometallic Reactions
}

Tom A. Young, Joseph J. Silcock, Alistair J. Sterling and Fernanda Duarte

Chemistry Research Laboratory, University of Oxford, Mansfield Road, Oxford OX1 3TA, U.K.

KEYWORDS: Transition states, Reaction mechanisms, Sampling, Molecules, Automation

\begin{abstract}
Calculating reaction profiles to aid in mechanistic elucidation has long been the domain of the expert computational chemist. Here, we introduce autodE, an open-source tool capable of locating transition states and minima and delivering a full reaction energy profile with minimal human effort (https://github.com/duartegroup/autodE). autodE is broadly applicable to study organic and organometallic reaction classes, including addition, substitution, elimination, migratory insertion, oxidative addition and reductive elimination; it accounts for conformational sampling of both minima and TSs, and is compatible with many electronic structure packages. The general applicability of autodE is demonstrated in complex multi-step reactions, including metal-catalyzed cobalt- and rhodium-catalyzed hydroformylation, and an Ireland-Claisen rearrangement.
\end{abstract}




\section{Introduction}

Automating the search for new chemical reactions is seen as one of the 'grand challenges' in computational chemistry. ${ }^{1,2}$ Discovering such thermodynamically and kinetically accessible reactions requires knowledge of the underlying potential energy surface (PES), which to a first approximation, involves the characterization of reactants, products, intermediates, and transition states (TSs). ${ }^{3-5}$ While this has been routine for computational chemists for decades, locating TSs remains time consuming and a highly non-systematic endeavour. ${ }^{6}$ To this end, many automated TS-search algorithms have been developed which may be broadly classified into single and double-ended methods. ${ }^{7,8}$ The former requires a single chemical structure from which TSs, intermediates, and products are located with no a priori information. Representative examples include the ab initio nanoreactor (AINR), ${ }^{9}$ which employs high temperature and pressure molecular dynamics (MD) simulations to identify chemical transformations; the single-ended growing-string method (SE-GSM), ${ }^{10}$ which iteratively generates structures along the PES and optimizes them until a TS and minima at each side of the string are found; the artificial force induced reaction (AFIR) method, ${ }^{11}$ which adds artificial external forces to the original PES of the target system, and the TSSCDS which uses high-energy dynamics simulations and a geometry-based algorithm to identify reactive pathways. ${ }^{12}$ While they allow for free exploration of unknown pathways, these methods remain costly and limited to small systems ( $\sim 10$ reactant atoms) if left to freely explore all transformations.

On the other hand, double-ended methods use knowledge of both reactants and products. Depending on the specific method either single or multiple steps can be studied. The latter uses reaction network enumeration algorithms, which allow for a systematic search of the reaction space; but hit an exponential wall of complexity if arbitrarily many intermediates are permitted. ${ }^{13,14}$ Single-step methods, although seemingly limited in scope, remain a powerful and affordable approach to explore many chemical processes determined by elementary steps. This is the case, for example, when the goal is to elucidate the origin of regio- or enantioselectivity or compare different synthetically-plausible pathways for a given transformation. Several methods have been developed following this philosophy, from linear and quasi-synchronous transit ${ }^{15,16}$ and related approaches ${ }^{17,18}$ to more recent developments based on growing ${ }^{19}$ and freezing string methods ${ }^{20,21}$ (GSM/FSM, Figure 1). Finally, heuristic approaches guided by either graph-based chemical rules or electronic-structure theory have been 
employed to explore a wide range of chemical reactions; including the Reaction Mechanism Generator ${ }^{22}$, which follows approaches pioneered by $\mathrm{NetGen}^{23}$, and other reaction network approaches. ${ }^{24,25}$
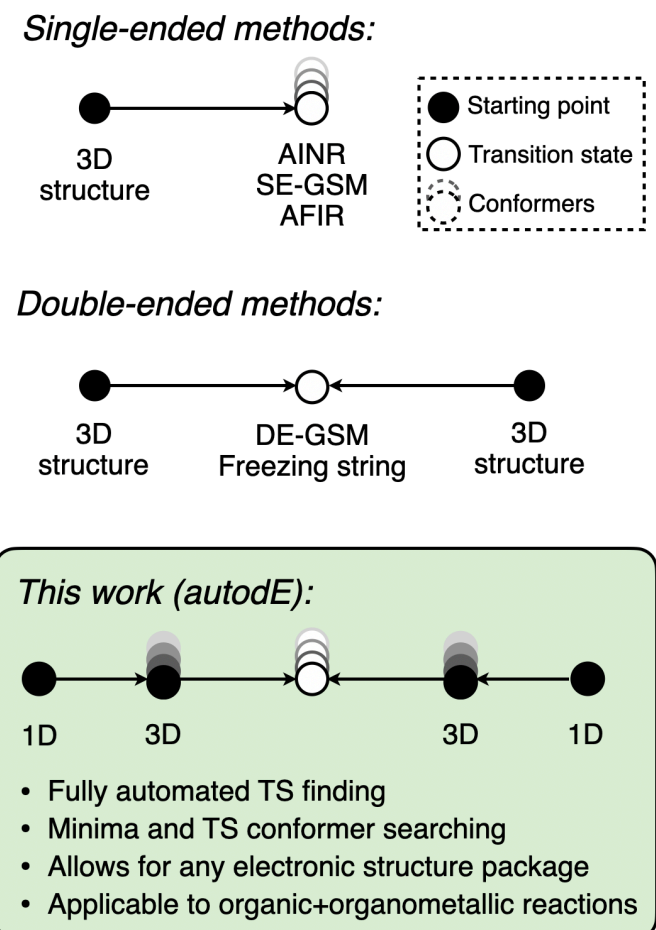

Figure 1. Comparison of approaches to generate reaction profiles.

Here, we introduce autodE, which combines elements of previously reported double-ended methods with the development of a general, easy to use, and freely available framework to automate the characterization of reaction pathways. The autodE algorithm takes inspiration from related methods, such as AutoTS ${ }^{26}$ developed by Schrodinger ${ }^{\circledR}$ and the Reaction Mechanism Generator developed by Green et al., ${ }^{22}$ and aims to overcome current limitations in applicability, conformational sampling and accessibility; specifically (1) it provides a broadly applicable framework to study organic and organometallic reaction classes; (2) it accounts for conformational sampling of both minima and transition states, which is essential particularly when exploring flexible systems; (3) it is freely available (open-source Python package under a MIT license) and requires minimal user input and expertise. Moreover, it is compatible with several electronic structure theory packages 
and extensible to others. This work describes the algorithm and implementation of autodE and demonstrates its capabilities in a range of representative organic and organometallic reactions. We demonstrate that autodE is capable of localizing the different TSs and minima along the PES and delivering a full reaction energy profile using only SMILES representations as inputs and with a minimal human effort. To illustrate the functionality and general applicability of autodE, we apply it to a range of reactions classes, including complex organic and metal-catalyzed reactions. 


\section{Methodology}

In general, human-guided characterization of TSs and reaction pathways requires: (1) locating reactants and products; (2) locating the TS, usually starting from a guess TS structure generated by chemical intuition; (3*) once reactants, TSs, and products have been characterized, perform a conformational search to identify the lowest energy conformer in each case; and $\left(4^{*}\right)$ performing single point energy evaluations to refine energies. While the starred steps are not always performed, they are usually necessary to achieve meaningful conclusion about the reactivity and selectivity of a given reaction step. Our method follows this workflow, which is described in detail in the following sections, along with representative examples (Figure 2).

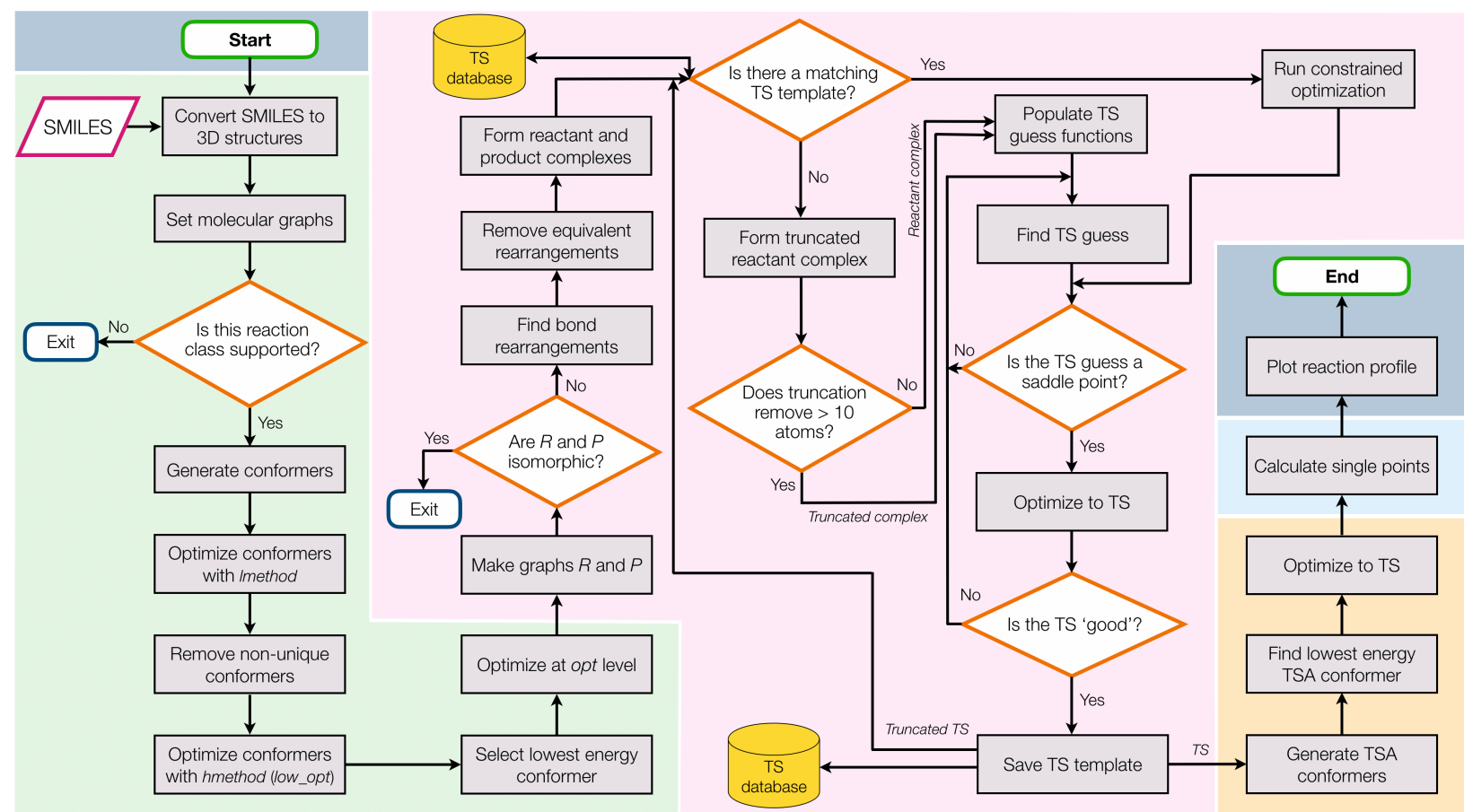

$\overline{\text { Figure 2. Diagrammatic workflow used by autodE to plot a reaction profile from a set of reactant(s) and product(s) }}$ SMILES strings. TSA $=$ transition state analogue; where active bonds are constrained at distances found at the TS found in the step above or saved in the TS database.

With a view to generating reaction profiles efficiently using currently available quantum mechanics methods, our implementation makes use of two levels of theory: a lower-level (lmethod) and higher-level method 
(hmethod). Both methods have default settings (Table 1) which can be modified by the user depending on the methods available in the respective software; for example, DFT methods can be used as lmethod for optimizations, and wavefunction methods as hmethod for single point energy evaluations. autodE is readily extensible to many other electronic structure theory codes and forcefields in addition to those with currently implemented wrappers (ORCA, ${ }^{27}$ NWChem, ${ }^{28}$ Gaussian09, ${ }^{29}$ MOPAC, ${ }^{30}$ GFN-XTB,${ }^{31}$ see discussion in the SI and Figure S1). In the following paragraphs we describe the key features and implementation of autodE.

Table 1. Default methods used in autodE calculations. $s p=$ single point energy, opt $=$ optimization, $l o w \_o p t=$ low level optimization, optts $=$ transition state optimization, hess $=$ Hessian. ${ }^{a}$. Uses D3 rather than D3BJ dispersion.

\begin{tabular}{ccc}
\hline Software Package & Calculation & Default Method \\
\hline ORCA, Gaussian09, NWChem ${ }^{a}$ & low_opt & PBE-D3BJ/def2-SVP \\
& opt, optts, hess & PBE0-D3BJ/def2-SVP \\
\hline MOPAC & $s p$ & PBE0-D3BJ/def2-TZVP \\
\hline XTB & low_opt, opt, sp & PM7 \\
\hline
\end{tabular}




\subsection{Finding Lowest Energy Conformers for Reactant and Products}

Locating the global minimum, or a structure close to it, on a PES is challenging but essential in estimating the feasibility of a reaction. ${ }^{32}$ To characterize the lowest energy conformer at a given level of theory requires transforming the 1D SMILES representation into a 3D geometry and searching the conformational space. Several open-source tools are available including $\mathrm{RDKit}^{33}$ and $\mathrm{CONFAB}^{34}$ implemented in OpenBabel, but are generally limited to organic species. autodE uses the ETKDGv2 ${ }^{35}$ algorithm implemented in RDKit, which is known to perform well for organic species, along with its SMILES parser. ${ }^{36}$ To determine optimal parameters for the number of conformers generated and the root mean squared (RMSD) threshold used to exclude identical conformers, a set of 20 amino acids were considered. Using $\mathrm{CREST}^{37}$ as the benchmark method, the generation of 600 conformers and RMSD threshold $=0.1 \AA$ was sufficient to obtain reliable minima, rendering a conformer within $1 \mathrm{kcal} \mathrm{mol}^{-1}$ of the most stable CREST conformer in 90\% of the cases (18/20) (Figure S2). Therefore, this inexpensive conformer search was kept as the default for organic molecules and allows for autodE to remain method agnostic (i.e lmethod/hmethod can be chosen by the user).

For metal complexes, where OpenBabel and RDKit fail to interpret the SMILES string and/or generate a sensible 3D geometry, we utilize our own SMILES parser and a simple repulsion+bonded (RB) forcefield (equation 1).

$$
U_{\mathrm{RB}}(\boldsymbol{x})=\sum_{i j \in \text { bonds }} k_{1}\left(r_{i j}-r_{i j}^{0}\right)^{2}+\sum_{i>j} \frac{k_{2}}{r_{i j}^{n}}
$$

The first harmonic term describes bonds between atoms, and the second term introduces a non-physical repulsion that enforces a notion of steric repulsion. Parameters $k_{1}=1$ and $k_{2}=0.01$ were selected based on empirical experience and comparison to optimized structures (Figure S3) while ideal bond lengths $\left(r^{0}\right)$ are obtained as averages from the Cambridge Structural Database. ${ }^{38}$

To generate reasonable 3D structures for organometallic complexes, each atom is added sequentially and randomly in a $10 \AA$ cubic box, and the function $U_{\mathrm{RB}}$ is minimized with respect to all coordinates after each addition. Using a smooth potential with few local minima, $n=2$ in Eq. 1, is required to obtain stable structures 
for large complexes (Figure S4). For a test set of 20 metal-complexes with up to 100 atoms, our approach delivers a stable conformer in all cases, while RDKit successfully generates only 15 geometries and CONFAB failed in all cases (Figure S5 and Table S1). With the analytic derivative of $U_{\mathrm{RB}}$ and a conjugate gradient algorithm implemented in $S c i P y,{ }^{39}$ an initial structure is available in a few seconds for complexes with more than 100 atoms. Stereodefined metal complexes are currently not supported, as random initialization does not respect any chirality.

An alternative and slightly faster approach used to generate conformers is to randomize all atoms by applying a random displacement vector (length $\sim 3 \AA$ ) and then minimize the function $U_{\mathrm{RB}}$ with $n=8$, as a more realistic surface (which is required to afford three minima in the butane dihedral PES, Figure S4). While simple, this strategy affords more conformers than both RDKit and CONFAB for the metal complex test set (Figure S5 and Table S1). It is also worth noting that we found no advantage in using the EMBED algorithm ${ }^{40}$ to generate initial coordinates for organic systems (Figure S6 and Table S2). Moreover, conformers can be generated using arbitrary distance constraints specified by the user (e.g. to retain a square planar geometry given an 3D initial structure).

In autodE, for either organic molecules or metal complexes, the lowest energy conformer is found by optimizing each conformer at the lmethod and excluding non-unique structures based on an energy threshold $\left(1 \mathrm{~kJ} \mathrm{~mol}^{-1}\right.$ default). The remaining structures are then optimized at the desired low_opt level with the hmethod, and the lowest energy is kept (Figure S6).

\subsection{TS Location}

Within autodE, each species has an associated molecular graph (X), in which the atoms correspond to vertices (V) and 'bonds' to edges (E). For a discussion of how bonds are defined, see the SI (Figure S7). From the set of reactant graphs $\left\{R_{i}\right\}$, the reactant graph $(R)$ is formed as the graph union, and likewise with products $\left\{P_{i}\right\}$ to generate $(P)$. This is represented in Figure 3 for a Claisen rearrangement, where the graphs $R$ and $P$ are formed from a single reactant and product molecule. If $R$ and $P$ are isomorphic then the chemical transformation is not obvious from the graph transformation and the program will exit. Once this has been cheeked, to find a atom 
mapping (bijection) from $R$ to $P$, we generate $\left\{R^{\prime}\right\}$, the set of transformations of $R$ obtained by adding and/or removing edges $\left[g: \mathrm{E}(R) \rightarrow \mathrm{E}\left(R^{\prime}\right)\right]$, where each function $\{g\}$ represents the bond rearrangement. The atom mapping(s) $\left[f: \mathrm{V}\left(R^{\prime}\right) \rightarrow \mathrm{V}(P)\right]$ are then found where $R^{\prime}$ and $P$ are isomorphic using NetworkX ${ }^{41}$. From this example, it is clear that a brute force search for all $g$ obtained by enumerating over all possible edge additions/deletions becomes intractable quickly, as the number of operations grows as $2^{N}$ for a system with $N$ atoms. However, chemical reactions usually involve a limited number of bonds that are formed and broken in a single elementary step, thereby substantially reducing the search space. We therefore limit the transformation to a maximum of four 'active' bonds where two edges are added and two removed, denoted $(2,2)$. For a graph $\mathrm{X}$ with $b$ bonds $\left(b_{X}=|\mathrm{E}(\mathrm{X})|\right)$ the principle of microscopic reversibility is used to switch reactants and products if $b_{\mathrm{R}}<b_{\mathrm{P}}$. From these constraints, five scenarios exist:

I. $\quad(1,1)-$ substitution reactions e.g. $\mathrm{S}_{\mathrm{N}} 2$.

II. $\quad(2,2)-$ substitution reactions e.g. peroxide epoxidation.

III. $(0,1)-$ dissociation e.g. E1 eliminations.

IV. $(0,2)-$ dissociations e.g. Diels-Alder cycloaddition.

V. $(1,2)-$ eliminations e.g. E2 eliminations.

Defining the change in the number of bonds as $\Delta b=b_{\mathrm{R}}-b_{\mathrm{P}}$ as in references ${ }^{26}$ and ${ }^{42}$, only when $\Delta b=0$ does the full set of deleting and adding two edges need to be enumerated. Further acceleration is achieved by calculating $\left\{\Delta b_{\mathrm{k}}\right\}$ where $k$ is a bond type e.g. ' $\mathrm{C}-\mathrm{C}$ ' and ensuring any atom (a) doesn't exceed a maximal valence i.e. $d(a) \leq 4$ for a carbon atom. For example, in a Claisen rearrangement $\Delta b_{\mathrm{C}-\mathrm{C}}=1$ and $\Delta b_{\mathrm{C}-\mathrm{O}}=-1$ such that the enumeration over $(1,1)$ transformations is targeted to only $\mathrm{C}-\mathrm{C}$ and $\mathrm{C}-\mathrm{O}$ bonds. Once the set of valid $\{g\}$ is found, a TS is located for each of them (if it exists), and following conformational sampling, the lowest energy TS is taken for the calculation of a reaction profile. For the Claisen reaction shown in Figure 3, only one bond rearrangement is obtained while for the all-carbon analogue $\left(\mathrm{O} \rightarrow \mathrm{CH}_{2}\right)$ two rearrangements are found $\left(g=\left\{g_{1}\right.\right.$, $\left.\left.g_{2}\right\}\right)$. We are yet to find a reaction where the process of generating/checking $R$ ' and $P$ isomorphisms and finding the bijection is comparable or more demanding than a DFT calculation. 


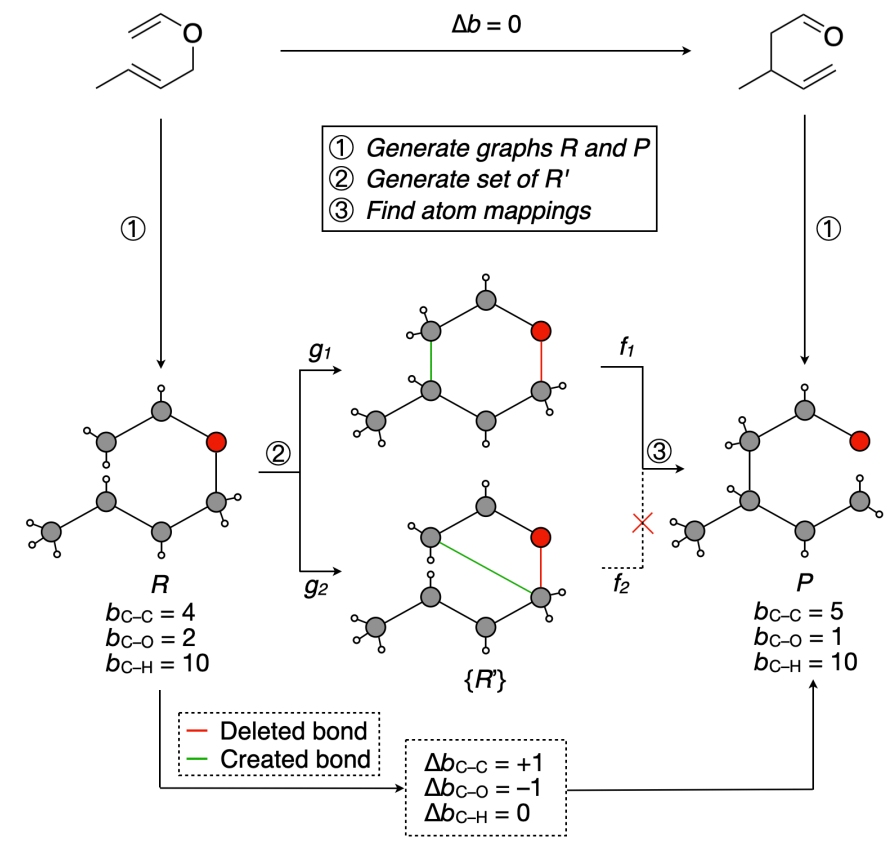

Figure 3. Atom mappings between reactants and products for a Claisen rearrangement.

To generate a saddle point, while ensuring compatibility with multiple electronic structure codes, we favor finding a TS guess by performing a set of constrained geometry optimizations along a linear path between reactants and products. In general, autodE attempts $1 \mathrm{D}$ constrained searches over forming/breaking bonds except when $\Delta b=2$ (e.g. Diels-Alder). If this procedure fails and the number of active bonds is more than one, a 2D PES exploration is performed by constrained optimizations and fitting a polynomial up to order $3 .{ }^{39}$ From a 2D PES, the lowest energy saddle point is found by locating where the first derivative vanishes, and then using Dijkstra's algorithm ${ }^{43}$ implemented in NetworkX. ${ }^{41}$ A constrained optimization at the saddle point is then performed to generate the TS guess. Once the TS guess is obtained, it is optimized with the TS optimizers implemented in the electronic structure theory package selected by the user. 'Goodness' of the TS is defined by an imaginary frequency mode $\left(\left|v_{\text {imag }}\right|>45 \mathrm{~cm}^{-1}\right)$ and a significant contribution from the active bonds, or forward/backward displacement along the mode affords reactants/products (a quick reaction coordinate, QRC calculation $^{44}$ ). In cases where the reactant or product(s) are not isomorphic to $R$ or $P$, conformers of the reactant and product complexes are generated (as outlined in \$2.6) and isomorphisms checked for the 
forwards/backwards displaced structures to all optimized conformers. This approach overcomes the limitation of NEB approaches that requires minimal structural difference between the initial and final states, which may be challenging when larger changes occur between the reactant and product complex or where the product is linked to $P$ by one or more barrierless reactions e.g. some proton transfers.

\subsection{Truncation}

Performing tens of constrained optimizations along one or two coordinates in a large system is currently computationally demanding. To accelerate the generation of a TS guess in such systems, the system is truncated. Our implementation truncates over $\mathrm{C}-\mathrm{X}$ bonds where the carbon atom is fully saturated and is at least two bonds away from the active atoms, and the truncated group is replaced by a hydrogen atom (Figure S8). The TS is then located using this truncated complex and saved in the template library from which the full non-truncated TS may be found using the templating method described below. For truncation to be utilized it must remove $>10$ atoms.

2.4. Finding Lowest Energy TS Conformers. TS conformers are located in a similar manner to the protocol described for metal complexes in $\mathbf{\$ 2 . 1}(n=8$ in Eq. 3 and randomization using a displacement vector of $\sim 3 \AA$ ). However, here the 'active' bonds are constrained at distances found at the TS using a harmonic potential with a force constant $k_{1}^{\prime}=10 k_{1}$ (equation 2).

$$
U_{\mathrm{RB}}{ }^{\prime}(\boldsymbol{x})=\sum_{i j \in \text { bonds }} k_{1}\left(r_{i j}-r_{i j}^{0}\right)^{2}+\sum_{i j \in \text { const. }} k_{1}^{\prime}\left(r_{i j}-r_{i j}^{0}\right)^{2}+\sum_{i>j} \frac{k_{2}}{r_{i j}^{8}}
$$

An identical method is then used to find the lowest energy transition state analogue (TSA) - as a model for the TS - performing optimizations with the active bonds constrained to their length in the TS. From the TSA a TS is found by rerunning the transition state optimization and checking that it is both a 'good' TS and is lower in energy than the previously found TS.

\subsection{Transition State Templates}

To accelerate the location of TSs, if available, a template is employed to generate a TS guess structure. Templates are saved into a library following the successful location of a TS and contain a graph, solvent, charge and 
multiplicity. For a template to be used, the reactant(complex) must have the same charge, multiplicity, and solvent parameters as the one used to obtain the template. In addition, the active bonds must match (same number and atoms) along with their nearest neighbors, based on their connectivity and atom type. If a matching template is found in the library, then a TS guess is generated by constrained optimization, where the active bonds are fixed at values found in the saved TS, from which TS optimization is performed. For example, a TS found for $\mathrm{EtCl}+\mathrm{F}^{-} \rightarrow \mathrm{EtF}+\mathrm{Cl}^{-}$enables a $10 \times$ faster characterization of the TS for the propyl analogue.

\subsection{Non-covalent Complexes}

In many chemical processes, the formation of a reactant/product complex precedes/follows the chemical step of interest, and are therefore fundamental to determine the kinetics of the process. In some cases, the molecular graph of the reactant and product complexes may not be isomorphic to the separated reactants $(R)$ and products $(P)$. For example, in the base-catalyzed hydrolysis of methyl acetate, loss of $\mathrm{MeO}^{-}$from the tetrahedral intermediate proceeds with concurrent deprotonation of the acid (Figure S9). Thus, to successfully characterize the TS as 'good', reactant and product complex conformers are required from which isomorphisms can be checked to the forward/reverse displaced species from the TS. This approach allows the lowest energy conformer of a non-covalent interaction (NCI) complex to be located systematically. For a complex formed by molecule A and $\mathrm{B}$, conformers of the A.B complex are constructed by adding molecule B to the current set of atoms of B recursively at points equally spaced on the surface of a sphere ${ }^{45}$ in a random orientation around A then energy minimizing (Figure S10). For a complex with $N_{R}$ reactants using $N_{\mathrm{s}}$ points on a sphere and $N_{\mathrm{r}}$ random rotations, this approach generates $\left(N_{\mathrm{s}} \times N_{\mathrm{r}}\right)^{N R-1}$ conformers. To maintain efficiency, a maximum threshold number of conformers (1000 default) is set, which are subsequently optimized using at least semiempirical or DFT as the lmethod. This approach also provides an additional functionality, e.g. facilitating the generation of hydrogen bond complexes of relevance in anion-recognition without prior knowledge (Figure S11).

\subsection{Reactive Reactant Complexes}

For bimolecular reactions (substitution and elimination, not addition as $b_{\mathrm{P}}>b_{\mathrm{R}}$ ) an initial NCI complex on the PES must be found that favors a linear arrangement of the nucleophile and the leaving group, while also reducing 
the required number of optimizations. For this reason, the energy function $U_{\mathrm{RR}}$ (equation 3 ) is minimized with respect to rigid-body rotation and translation of one reactive component with empirical parameters $c_{1}=c_{2}=c_{3}$ $=1, c_{4}=10$ and $c_{5}=2.5(1.5)$ for charged (uncharged) complexes.

$$
U_{\mathrm{RR}}\left(\boldsymbol{x}_{i}\right)=\sum_{\{\mathrm{ac}\}} c_{1}\left(r_{\mathrm{ac}}-c_{5} r_{\mathrm{ac}}^{\mathrm{avg}}\right)^{2}+\sum_{\{\mathrm{acx}\}} c_{2}(1-\cos \theta)+\sum_{\{\mathrm{acx}\}} c_{3}(1-\cos \phi)+\sum_{\{\mathrm{ij}\}} \frac{c_{4}}{r_{\mathrm{ij}}^{4}}
$$

This energy function has been designed to maximize the collinearity of vectors $\boldsymbol{v}_{\mathrm{an}}, \boldsymbol{v}_{\mathrm{ac}}$ and $\boldsymbol{v}_{\mathrm{cx}}$ while maintaining both a low steric repulsion and distance $\left(r_{\mathrm{ac}}=\left|\boldsymbol{v}_{\mathrm{ac}}\right|\right)$ close to that desired (Figure 4). This choice is guided by chemical intuition, and there are rare cases where this does not adhere to e.g. front-side $\mathrm{S}_{\mathrm{N}} 2{ }^{1}{ }^{46}$ For example, for $\mathrm{S}_{\mathrm{N}} 2$ substitution, E2 elimination and $\mathrm{S}_{\mathrm{N}} \mathrm{Ar}$ reactions adequate initial geometries are obtained (Figure 4, S12). Note that there may be several minima on this surface; thus, the multiple initial random rotations and translations $(\sim 10)$ are performed to have a better chance of locating the global minimum while remaining computationally inexpensive (execution time $\sim$ seconds).

A front side $\mathrm{S}_{\mathrm{N}} 2$ mechanism is, however, found when conformers of a backside $\mathrm{S}, 2 \mathrm{TS}$ are generated. 

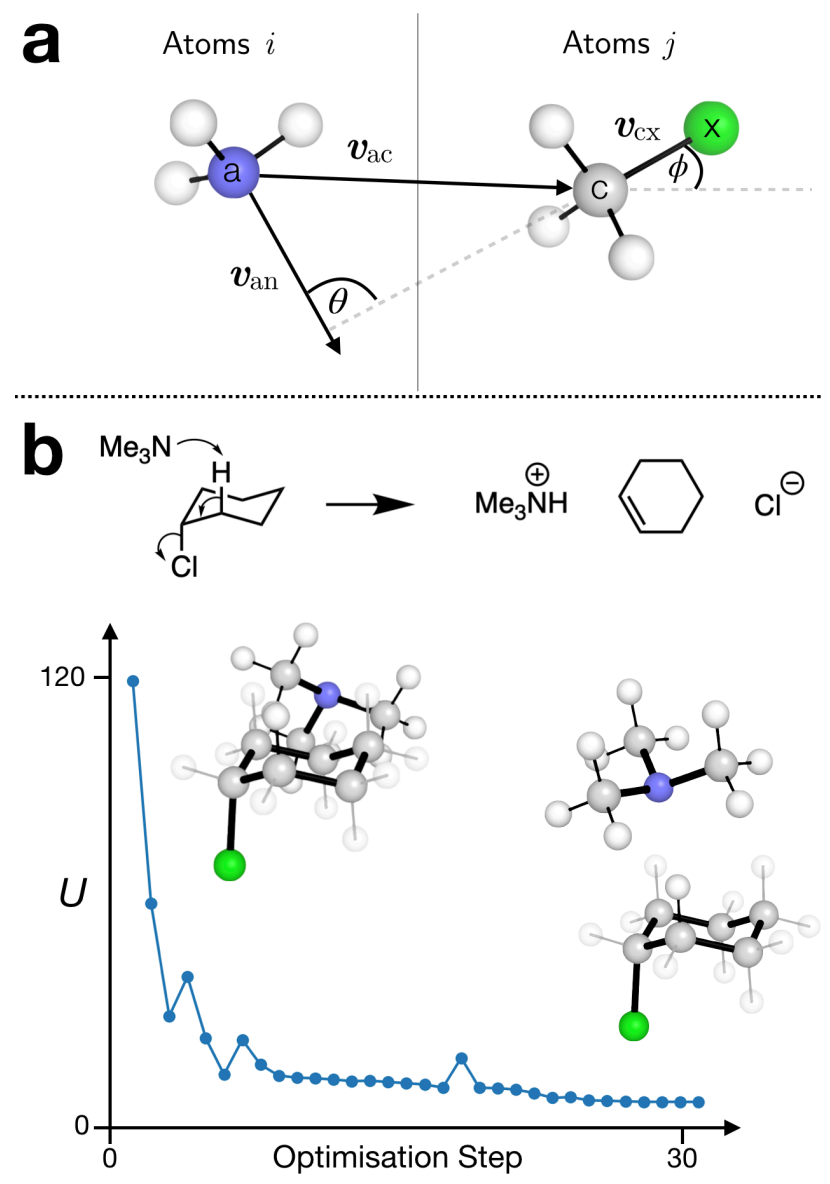

Figure 4. (a) Illustration of the geometric parameters used in equation 2 using the $\mathrm{S}_{\mathrm{N}} 2$ reaction $\left(\mathrm{NH}_{3}+\mathrm{CH}_{3} \mathrm{Cl}\right)$ as an example: $\boldsymbol{v}_{\mathrm{an}}$ is the average of vectors $\left\{\boldsymbol{v}_{\mathrm{am}}\right\}$ where $m$ is a nearest neighbor to $a$. (b) Minimization of the function $U\left(x_{i}\right)$ for an E2 reaction; the energy profile is qualitative as it depends on an initial random configuration. Initial and optimized structures are shown. 


\section{Results and Discussion}

To demonstrate the applicability of autodE in multiple reaction classes, we explored some textbook organic reactions $\left(\mathrm{S}_{\mathrm{N}} 2, \mathrm{E} 2\right.$, etc.) alongside industrially relevant organic and metal-catalyzed reactions involving more than 50 atoms. By way of example, we demonstrate that autodE is broadly applicable, efficient in exploring conformational space, and straightforward to use.

Even with a small amount of programming experience using autodE is as simple as declaring reactants and products involved in the reaction from their respective SMILES strings (or from xyz files) and optionally implicit solvent. To calculate the reaction profile for a simple $S_{N} 2$ reaction the input and output are shown in Figure 5, where the calculation is parallelized over four processing cores, the reaction immersed in an implicit aqueous environment and reactant/product are defined as SMILES strings. Here, by calling the method calculate_reaction_profile(with_complexes=True), the association complexes are also calculated along the energy profile. Alternatively, one can decide only to obtain the TS, with the function locate_transition_state. 

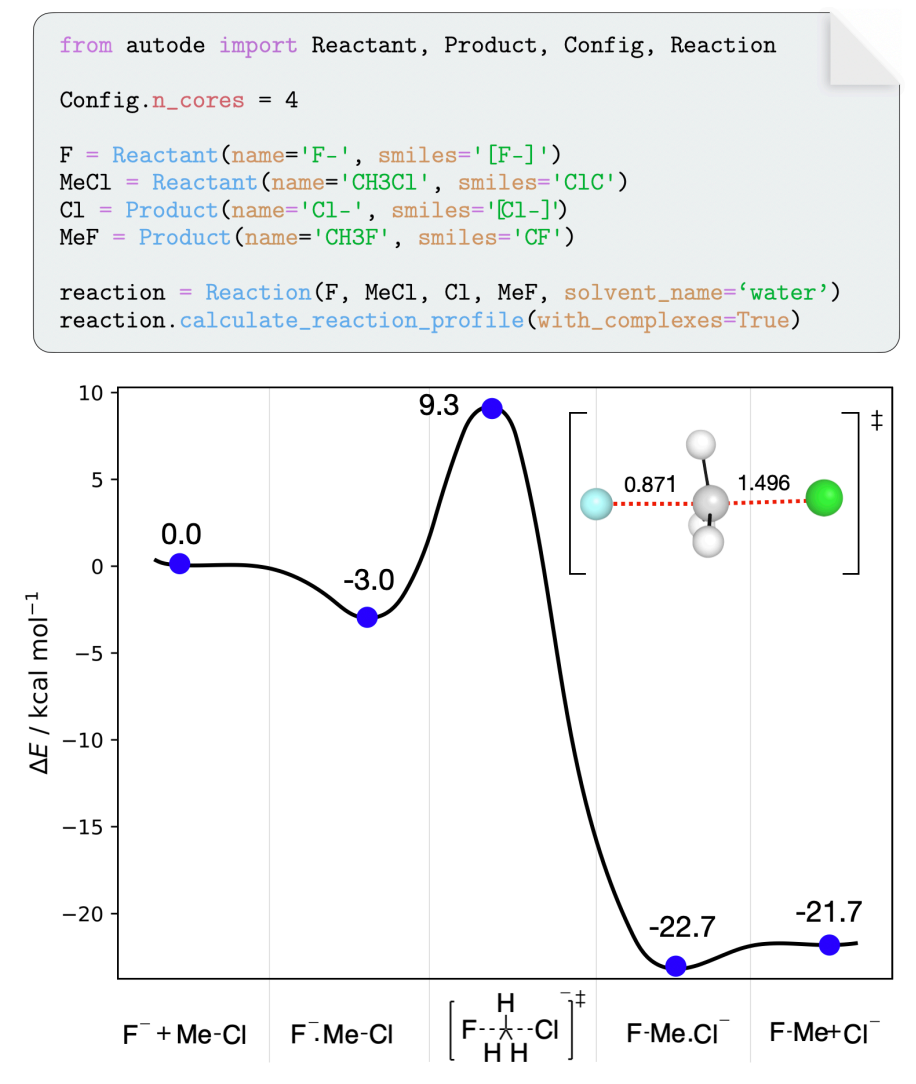

$\overline{\text { Figure 5. Reaction energy profile for an } \mathrm{S}_{\mathrm{N}} 2 \text { reaction calculated using autodE. Electronic structure calculations were }}$ performed at the GFN2-XTB and DFT level in XTB/ORCA, respectively, for geometry optimization/energy calculations. Final energies quoted at the $\mathrm{CPCM}\left(\mathrm{H}_{2} \mathrm{O}\right)-\mathrm{PBE} 0-\mathrm{D} 3 \mathrm{BJ} / \mathrm{def} 2-\mathrm{TZVP} / / \mathrm{CPCM}\left(\mathrm{H}_{2} \mathrm{O}\right)-\mathrm{PBE} 0-\mathrm{D} 3 \mathrm{BJ} / \mathrm{def} 2-$ SVP level of theory. Distances in $\AA$.

Without specifying which electronic structure codes to use, autodE employs first available hmethod and lmethod, (sequentially ORCA, Gaussian09, NWChem and XTB, MOPAC respectively), with the default DFT methodologies listed in Table 1. Using this setup, the reaction energy profile shown in Figure 5 is obtained in less than 10 minutes. While it would be ideal to obtain reaction free energies, the cost and limitations associated with the calculation of entropy and thermal contributions using currently available models (ideal gas) mean that the addition of such corrections should be considered with care. For this reason, potential energies are set as the default in autodE. However, free energies can be obtained by running frequency calculations which can be achieved within a Python environment using autodE by performing a Hessian calculation (with e.g. 
Calculation(molecule, method, keywords=method. keywords.hess, ..., see the documentation for examples: https://duartegroup.github.io/autodE/).

\subsection{TS Conformers}

A thorough exploration of the conformational space in order to find the lowest energy conformer for reactants and TSs is essential in characterizing the kinetic and thermodynamic parameters of a reaction. As discussed in §2.2, autodE provides two routes to locating TS conformers: The first uses reactant/product complex conformers, from which different TSs may be traversed, and the second locates TS conformers directly. If using templates, both approaches are efficient, generally requiring only one constrained optimization and one TS optimization per conformer once a TS has been found. Direct TS conformer searching is, however, faster as only the TSAs are optimized and the lowest energy found whereas full enumeration from reactant/product complexes can require rescanning over the PES.

For example, for an E2 elimination reaction, the initial search may locate a transition state in which the deprotonation occurs with the proton and the leaving group in a syn conformation, rather than the favored anti conformer. The latter TS is automatically found in autodE by employing the strategy outlined in \$2.2 (Figure 6a). Similarly, the autodE algorithm correctly locates the lowest energy H-migration TS for the radical decomposition of 1-propanol, with an RMSD $<0.1$ Å compared to the human-generated TS from ref ${ }^{47}$ (Figure S13). The importance of this unbiased TS conformer generation is highlighted in the Michael addition of nitromethyl acetate and methyl vinyl ketone, where several rotable bonds exist (Figure 6b). For this reaction, an exhaustive search from product conformers generated $21 \mathrm{TS}$ conformers, which upon optimization, led to a range in activation energies of more than $5 \mathrm{kcal} \mathrm{mol}^{-1}$ and reaction energies that differ by up to $17 \mathrm{kcal} \mathrm{mol}^{-1}$. The weak correlation between $\Delta E^{*}$ and $\Delta E_{\mathrm{r}}$ highlights the importance of a systematic conformer search at both minima and transition states. 


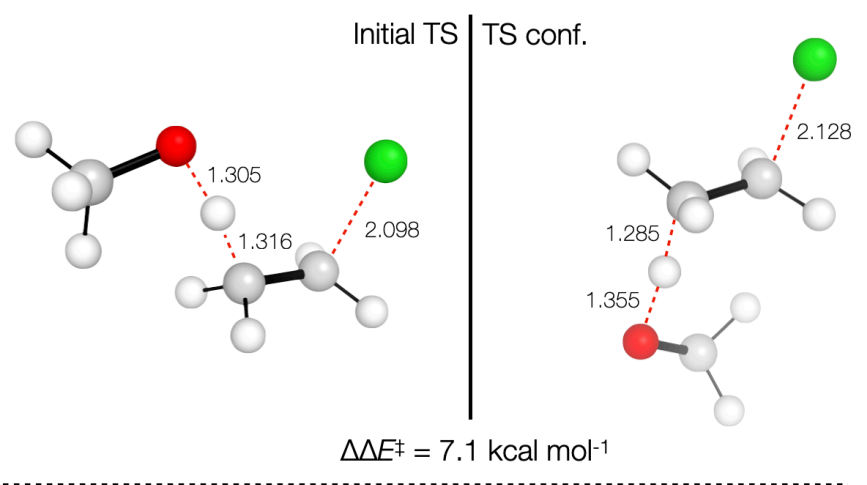

b TS conformers from product conformers

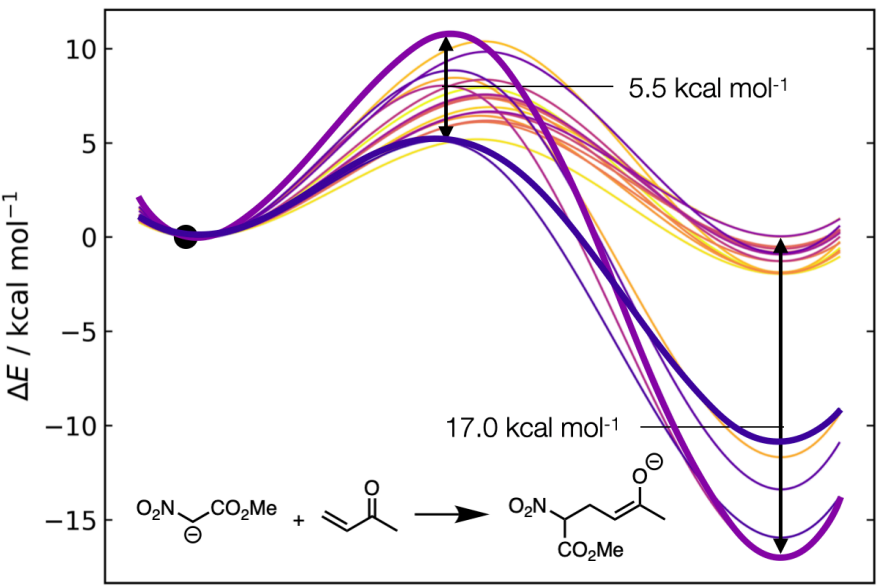

Figure 6. (a) Lowest energy transition state conformers found with autodE for methoxide + chloroethane elimination.

(b) TS and product conformer distributions for the reaction between nitromethyl acetate and methyl vinyl ketone addition.

\subsection{Organic Reaction}

For autodE to become routinely used in mechanistic investigations, it must be applicable to synthetically relevant, and usually more complex, reactions. Here, we explored the unexpected Ireland-Claisen rearrangement of divinylcyclopropanes reported by the Stoltz group and explored computationally via trial-and-error (Houk group) and the AFIR method. ${ }^{48}$ With known intermediates, processed as SMILES strings from Chemdraw ${ }^{\circledR}$, autodE was tested and compared to the human guided approach, which originally involved the testing of various conceivable mechanisms. autodE delivered an essentially identical reaction profile and reduced the time from the year of human and compute effort reported in ref 47 to a few minutes of setup time and $\sim 600$ CPUh (one day 
of computer time in 24 cores) (Figure 7). Interestingly, autodE deviates $<2 \mathrm{kcal} \mathrm{mol}^{-1}$ from the trial and errorbased search (blue), with INT3 showing a larger difference of $5 \mathrm{kcal} \mathrm{mol}^{-1}$ due to autodE finding a more stable than the one located previously.

This example demonstrates that a compilation of chemical knowledge, required to hypothesise reasonable mechanisms, and the use of autodE, can substantially speed up joint experimental and computational efforts to elucidate complex reaction mechanisms, and in this way advance the optimisation of synthetic routes and the design of novel catalysts.

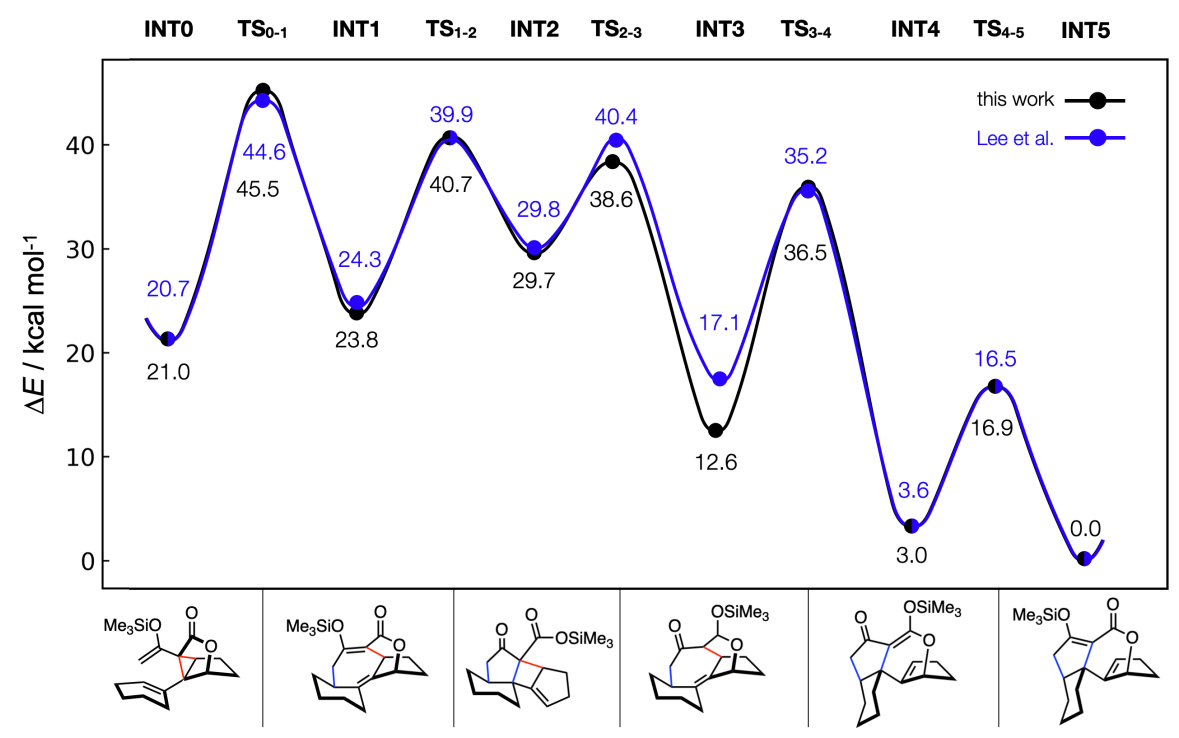

Figure 7. Ireland-Claisen rearrangement calculated using autodE (black) and described in ref ${ }^{48}$ (blue) calculated at B3LYP-D3BJ/6-311++G(2d,2p)// B3LYP-D3BJ/6- 31G(d). CPCM(hexane) solvent model used in ORCA (this work) and IEF-PCM(hexane) in Gaussian09 (ref. ${ }^{48}$ ). 3D Structures of the most stable intermediates and transition states are show in Figure S14.

\subsection{Organometallic Reaction}

The conversion of alkenes into aldehydes via addition of $\mathrm{CO}$ and $\mathrm{H}_{2}$ is an exceptionally important industrial process, making catalyst optimization the subject of considerable study. ${ }^{49}$ The mechanism was discovered by Heck and Breslow ${ }^{50}$ and has been the subject of numerous computational studies (for an overview of these studies see ref ${ }^{51}$ and references cited therein). Those works have provided significant insights into the 
mechanism of the reaction, and have also highlighted the challenges associated with it. In fact, only finding the intermediates and transition states along the PES is already a laborious process, requiring extensive sampling, due to the presence of several conformers and isomeric forms (Figure 8a).

Applying autodE to study this catalytic cycle using only the SMILES strings of the catalyst, ethylene, intermediates and products, the full reaction profile was obtained in just under 26 hours (16 CPU cores, excluding electronically barrierless ligand association steps (Figure 8b).
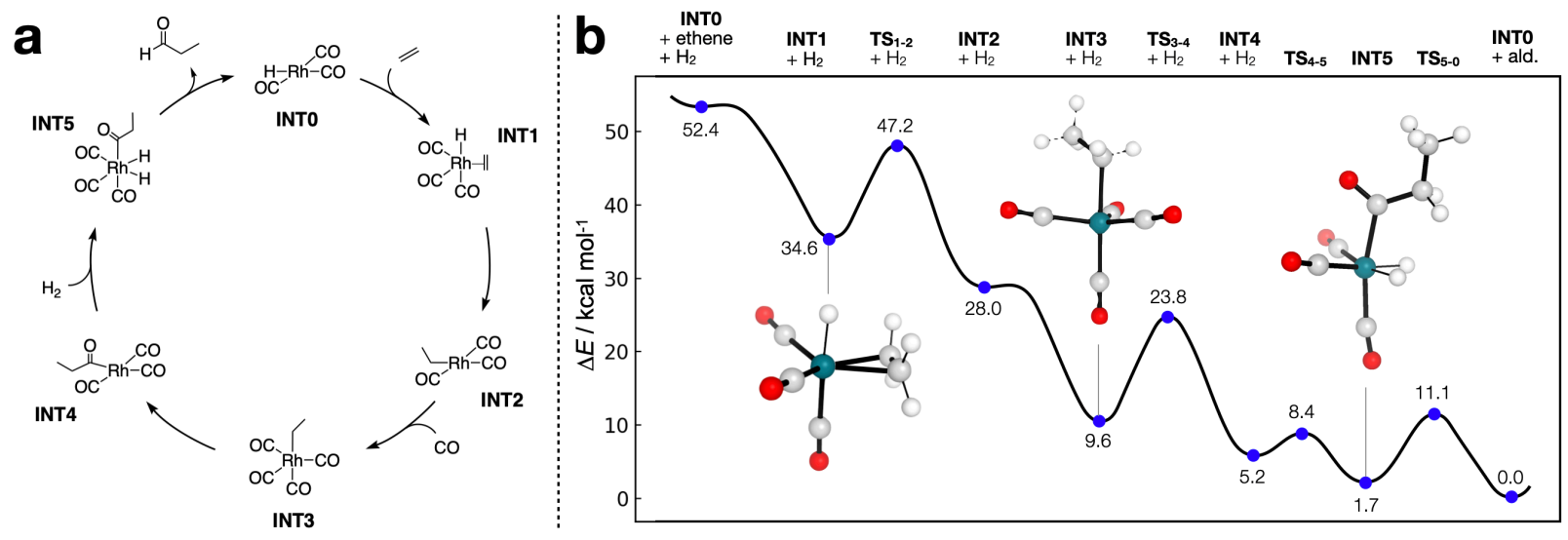

Figure 8. (a) Heck-Breslow mechanism of hydroformylation in the absence of a phosphine catalyst. (b) Reaction profile calculated at PBE0-D3BJ/def2-TZVP//PBE0-D3BJ/def2-SVP in autodE.

autodE correctly identifies the most stable isomer of all intermediates, for example the axial isomer for strong $\sigma$-donors (H: INT1, alkyl: INT3, acyl: INT5). TSs for all steps in the cycle are located successfully, contain a single imaginary frequency and have geometries very similar to those found for analogous phosphine-based catalysts (Figure S15). ${ }^{52}$ The same process was applied to the analogous Co-catalyzed hydroformylation (Figure S16), which has been used as a representative example in other automated reaction generation algorithms. ${ }^{13,14,53}$ Once again, all TSs were successfully located with minutes of setup time.

With a view on expanding the use of autodE for catalyst design campaigns, and following previous efforts in this field, ${ }^{54}$ we have also developed the molfunc tool (https://github.com/duartegroup/molfunc). Combining autodE with molfunc enables a rapid screening of different catalysts by performing functionalization of 
hydrogenic or other monovalent atomic positions. For example, for the H-insertion step, molfunc facilitates the exploration of different groups at the para position of phosphine on the barrier (Figure S17). The obtained positive correlation between the enhanced electron withdrawing ability of the catalyst and rate agrees with the experimental observation. ${ }^{51}$

\subsection{Further Examples}

To demonstrate the generality of our method, we tested autodE on a set of 15 simple organic reactions of different classes. autodE finds all TSs in all cases and generates reactions profiles in a matter of hours (Figure S18). Further examples include metal-catalyzed carbonylation of methanol to generate acetic acid via the Monsanto (Rh, Figure S19-20) and Cativa (Ir, Figure S21-22) processes,${ }^{55}$ alkaline ester hydrolysis (Figure S9), a HoukList TS (Figure S23), ${ }^{56}$ and 57 carbene insertion reactions reported in ef $^{57}$ (Figure S24). In this latter case autodE correctly locates 55/57 insertion TSs, with two failures occurring when a QRC+graph isomorphism check fails to confirm the TS as linking reactants and products, despite the TS being correct. Moreover, TSs for synthetically relevant reactions including a key Diels-Alder step in a total synthesis of Brevianamide A investigated by Domingo et al. ${ }^{58}$ and a diastereoselective epoxidation ${ }^{59}$ are presented in the SI (Figure S25-26).

\subsection{Limitations}

The graph-based approach affords two immediate limitations of the method: The need to define bonds, and checking stereochemistry. While bonds are generally well-defined in organic systems, they are not a rigid concept. Indeed, the energy required to overcome an interaction between atoms (i.e. a bond) does not necessarily correlate with the type of interaction (e.g. covalent or non-covalent) (Figure S27). This is partially alleviated by not regenerating the molecular graphs from the 3D structure and retaining the SMILES-defined connectivity but may afford an incorrect assignment of a 'good' TS, which actually does or does not lead to products/reactants. Furthermore, the isomorphism condition on reactants/products does not currently include a stereochemistry check - the algorithm simply finds the lowest energy TS. While this is often defined by the reaction type e.g. stereochemical inversion in $\mathrm{S}_{\mathrm{N}} 2$ is generated by traversing the lowest energy TS there are reactions such as the Peterson olefination where this is not adhered to. 
By enforcing a maximum connectivity change of four (up to 2 bonds breaking and forming) means that, for example, the TS in a synchronous Fritsch-Buttenberg-Wiechell rearrangement ${ }^{60}$ mechanism cannot be found. In this case, chemical intuition is required to prevent the very slow enumeration of 10 2D PES scans if all combinations of coordinates are chosen i.e. here some human intervention is necessary.

Finally, we note a limitation in the current lmethod electronic structure theory methods used to generate conformers of reactant and product complexes. Reactions involving anions are particularly problematic, as at the TB-DFT level they are generally not sufficiently stable (even in implicit solvent) such that the reaction may be barrierless at the lmethod level. We hope that as semi-empirical/TB electronic structure methods become more accurate and/or DFT and WF methods become faster, this limitation will be mitigated. Because autodE does not rely on one specific method implementing novel methods should be only an afternoons work.

\section{Conclusion}

Converting a $2 \mathrm{D}$ representation of a reaction to a reaction profile has long been the domain of expert computational chemists. Here we have shown that our method, autodE, can largely automate this process and present a selection of both simple and synthetically interesting organic and organometallic reactions. By building autodE in a way that does not rely on a particular electronic structure theory method, future methods for calculating points on the potential energy surface are easy to include. Indeed, the dominant source of failure of the methodology is due to inaccuracies in electronic structure methods rather than issues associated with autodE. Crucially, autodE is open source, making the development faster, more collaborative and more robust. We believe autodE will facilitate faster computational investigation. 
Computational Methods. All autodE calculations were performed with a development version of the code with the beta release distributed with this manuscript. Unless otherwise stated the lmethod used was GFN2-xTB v. 6.2 ${ }^{31}$ and the hmethod ORCA v. 4.2.27 All ORCA optimizations employed resolution of identify DFT (RI or RIJCOSX for hybrid functionals $)^{\alpha, 2}$ using the $\mathrm{PBE}^{63}$ or PBE0 functional, in combination with the D3BJ

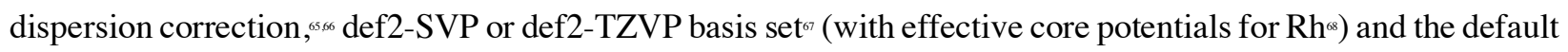
auxiliary basis set.9 Gaussian099 calculations employed identical methods without density fitting unless otherwise specified. NWChem v. 6.6 $6^{28}$ calculations used no dispersion correction as D3BJ is unavailable with PBE0. Default integration grids were used in all DFT calculations. Conformers generated with RDKit ${ }^{3}$ version 2020.03.1 and OpenBabel version 2.4.1.

Supporting Information. Compatibility of autodE with different electronic structure theory codes; details on the cconstruction of molecular graphs, evaluation of conformer generation approaches, and further examples on the applicability of autodE on organic and organomatalic processes.

Corresponding Author: FD: fernanda.duartegonzalez@chem.ox.ac.uk

Author Contributions: : T.A.Y and J. S developed the methodology and wrote the code. T. A. Y, J. S and A. J. S tested the package. F. D and T. A. Y wrote the manuscript with contributions from all authors.

Acknowledgments: We acknowledge the EPSRC Centre for Doctoral Theory and Modelling in Chemical Sciences (EP/L015722/1) for a studentship to T.A.Y. generously supported by AWE and for access to the Dirac cluster at Oxford. F.D. thanks the Carnegie Trust (RIG007448) and the John Fell Oxford University Press Research Fund (0006752) for financial support. A. J. S. thanks the EPSRC Centre for Doctoral Training in Synthesis for Biology and Medicine for a studentship (EP/L015838/1), generously supported by AstraZeneca, Diamond Light Source, Defence Science and Technology Laboratory, Evotec, GlaxoSmithKline, Janssen, Novartis, Pfizer, Syngenta, Takeda, UCB and Vertex. A. J. S. also thanks the Oxford-Radcliffe Scholarship for a studentship. 


\section{References}

(1) Grimme, S.; Schreiner, P. R. Computational Chemistry: The Fate of Current Methods and Future Challenges. Angew. Chemie Int. Ed. 2018, 57 (16), 4170-4176.

(2) Foscato, M.; Jensen, V. R. Automated in Silico Design of Homogeneous Catalysts. ACS Catal. 2020, 10 (3), 2354-2377.

(3) Cheng, G.-J.; Zhang, X.; Chung, L. W.; Xu, L.; Wu, Y.-D. Computational Organic Chemistry: Bridging Theory and Experiment in Establishing the Mechanisms of Chemical Reactions. J. Am. Chem. Soc. 2015, 137 (5), 1706-1725.

(4) Sameera, W. M. C.; Maseras, F. Transition Metal Catalysis by Density Functional Theory and Density Functional Theory/Molecular Mechanics. Wiley Interdiscip. Rev. Comput. Mol. Sci. 2012, 2 (3), 375-385.

(5) Kozuch, S.; Shaik, S. How to Conceptualize Catalytic Cycles? The Energetic Span Model. Acc. Chem. Res. 2011, 44 (2), 101-110.

(6) Simm, G. N.; Vaucher, A. C.; Reiher, M. Exploration of Reaction Pathways and Chemical Transformation Networks. J. Phys. Chem. A 2019, 123 (2), 385-399.

(7) Jensen, K. F.; Coley, C. W.; Eyke, N. S. Autonomous Discovery in the Chemical Sciences Part I: Progress. Angew. Chemie Int. Ed. 2020.

(8) Dewyer, A. L.; Argüelles, A. J.; Zimmerman, P. M. Methods for Exploring Reaction Space in Molecular Systems. Wiley Interdiscip. Rev. Comput. Mol. Sci. 2018, 8 (2), e1354.

(9) Wang, L.-P.; Titov, A.; McGibbon, R.; Liu, F.; Pande, V. S.; Martínez, T. J. Discovering Chemistry with an Ab Initio Nanoreactor. Nat. Chem. 2014, 6 (12), 1044-1048.

(10) Zimmerman, P. M. Single-Ended Transition State Finding with the Growing String Method. J. Comput. Chem. 2015, 36 (9), 601-611.

(11) Maeda, S.; Harabuchi, Y.; Takagi, M.; Taketsugu, T.; Morokuma, K. Artificial Force Induced Reaction (AFIR) Method for Exploring Quantum Chemical Potential Energy Surfaces. Chem. Rec. 2016, 16 (5), 22322248.

(12) Martínez-Núñez, E. An Automated Method to Find Transition States Using Chemical Dynamics Simulations. J. Comput. Chem. 2015, 36 (4), 222-234.

(13) Kim, Y.; Kim, J. W.; Kim, Z.; Kim, W. Y. Efficient Prediction of Reaction Paths through Molecular Graph and Reaction Network Analysis. Chem. Sci. 2018, 9 (4), 825-835.

(14) Habershon, S. Automated Prediction of Catalytic Mechanism and Rate Law Using Graph-Based Reaction Path Sampling. J. Chem. Theory Comput. 2016, 12 (4), 1786-1798.

(15) Halgren, T. A.; Lipscomb, W. N. The Synchronous-Transit Method for Determining Reaction Pathways and Locating Molecular Transition States. Chem. Phys. Lett. 1977, 49 (2), 225-232.

(16) Peng, C.; Bernhard Schlegel, H. Combining Synchronous Transit and Quasi-Newton Methods to Find Transition States. Isr. J. Chem. 1993, 33 (4), 449-454.

(17) Dewar, M. J. S.; Healy, E. F.; Stewart, J. J. P. Location of Transition States in Reaction Mechanisms. J. Chem. Soc. Faraday Trans. 2 1984, 80 (3), 227.

(18) Elber, R.; Karplus, M. A Method for Determining Reaction Paths in Large Molecules: Application to Myoglobin. Chem. Phys. Lett. 1987, 139 (5), 375-380.

(19) Dohm, S.; Bursch, M.; Hansen, A.; Grimme, S. Semiautomated Transition State Localization for Organometallic Complexes with Semiempirical Quantum Chemical Methods. J. Chem. Theory Comput. 2020, 16 (3), 2002-2012.

(20) Suleimanov, Y. V.; Green, W. H. Automated Discovery of Elementary Chemical Reaction Steps Using Freezing String and Berny Optimization Methods. J. Chem. Theory Comput. 2015, 11 (9), 4248-4259.

(21) Behn, A.; Zimmerman, P. M.; Bell, A. T.; Head-Gordon, M. Efficient Exploration of Reaction Paths via a Freezing String Method. J. Chem. Phys. 2011, 135 (22), 224108.

(22) Gao, C. W.; Allen, J. W.; Green, W. H.; West, R. H. Reaction Mechanism Generator: Automatic Construction of Chemical Kinetic Mechanisms. Comput. Phys. Commun. 2016, 203, 212-225.

(23) Broadbelt, L. J.; Stark, S. M.; Klein, M. T. Computer Generated Pyrolysis Modeling: On-the-Fly Generation of Species, Reactions, and Rates. Ind. Eng. Chem. Res. 1994, 33 (4), 790-799.

(24) Rappoport, D.; Aspuru-Guzik, A. Predicting Feasible Organic Reaction Pathways Using Heuristically Aided Quantum Chemistry. J. Chem. Theory Comput. 2019, 15 (7), 4099-4112.

(25) Bergeler, M.; Simm, G. N.; Proppe, J.; Reiher, M. Heuristics-Guided Exploration of Reaction Mechanisms. J. Chem. Theory Comput. 2015, 11 (12), 5712-5722. 
(26) Jacobson, L. D.; Bochevarov, A. D.; Watson, M. A.; Hughes, T. F.; Rinaldo, D.; Ehrlich, S.; Steinbrecher, T. B.; Vaitheeswaran, S.; Philipp, D. M.; Halls, M. D.; Friesner, R. A. Automated Transition State Search and Its Application to Diverse Types of Organic Reactions. J. Chem. Theory Comput. 2017, 13 (11), 5780-5797.

(27) Neese, F. Software Update: The ORCA Program System, Version 4.0. WIREs Comput. Mol. Sci. 2018, 8 (1), $1-6$.

(28) Valiev, M.; Bylaska, E. J.; Govind, N.; Kowalski, K.; Straatsma, T. P.; Van Dam, H. J. J.; Wang, D.; Nieplocha, J.; Apra, E.; Windus, T. L.; de Jong, W. A. NWChem: A Comprehensive and Scalable OpenSource Solution for Large Scale Molecular Simulations. Comput. Phys. Commun. 2010, 181 (9), 1477-1489.

(29) Frisch, M. J.; Trucks, G. W.; Schlegel, H. B.; Scuseria, G. E.; Robb, M. A.; Cheeseman, J. R.; Scalmani, G.; Barone, V.; Petersson, G. A.; Nakatsuji, H.; Li, X.; Caricato, M.; Marenich, A. V.; Bloino, J.; Janesko, B. G.; Gomperts, R.; Mennucci, B.; Hratchian, H. P.; Ortiz, J. V.; Izmaylov, A. F.; Sonnenberg, J. L.; WilliamsYoung, D.; Ding, F.; Lipparini, F.; Egidi, F.; Goings, J.; Peng, B.; Petrone, A.; Henderson, T.; Ranasinghe, D.; Zakrzewski, V. G.; Gao, J.; Rega, N.; Zheng, G.; Liang, W.; Hada, M.; Ehara, M.; Toyota, K.; Fukuda, R.; Hasegawa, J.; Ishida, M.; Nakajima, T.; Honda, Y.; Kitao, O.; Nakai, H.; Vreven, T.; Throssell, K.; Montgomery, J. A., J.; Peralta, J. E.; Ogliaro, F.; Bearpark, M. J.; Heyd, J. J.; Brothers, E. N.; Kudin, K. N.; Staroverov, V. N.; Keith, T. A.; Kobayashi, R.; Normand, J.; Raghavachari, K.; Rendell, A. P.; Burant, J. C.; Iyengar, S. S.; Tomasi, J.; Cossi, M.; Millam, J. M.; Klene, M.; Adamo, C.; Cammi, R.; Ochterski, J. W.; Martin, R. L.; Morokuma, K.; Farkas, O.; Foresman, J. B.; Fox, D. J. Gaussian 09, Revision A.02. Gaussian, Inc.: Wallingford CT 2016.

(30) Stewart, J. J. P. MOPAC2016; Stewart Computational Chemistry: Colorado Springs, CO, USA, 2016.

(31) Bannwarth, C.; Ehlert, S.; Grimme, S. GFN2-XTB - An Accurate and Broadly Parametrized Self-Consistent Tight-Binding Quantum Chemical Method with Multipole Electrostatics and Density-Dependent Dispersion Contributions. J. Chem. Theory Comput. 2019, 15 (3), 1652-1671.

(32) Chan, L.; Hutchison, G. R.; Morris, G. M. Bayesian Optimization for Conformer Generation. J. Cheminform. 2019, 11 (1), 32.

(33) Landrum, G. RDKit: Open-Source Cheminformatics, v. 2019; GitHub (https://github.com/rdkit/rdkit), 2019.

(34) O’Boyle, N. M.; Vandermeersch, T.; Flynn, C. J.; Maguire, A. R.; Hutchison, G. R. Confab - Systematic Generation of Diverse Low-Energy Conformers. J. Cheminform. 2011, 3 (1), 8.

(35) Riniker, S.; Landrum, G. A. Better Informed Distance Geometry: Using What We Know To Improve Conformation Generation. J. Chem. Inf. Model. 2015, 55 (12), 2562-2574.

(36) Ebejer, J.-P.; Morris, G. M.; Deane, C. M. Freely Available Conformer Generation Methods: How Good Are They? J. Chem. Inf. Model. 2012, 52 (5), 1146-1158.

(37) Pracht, P.; Bohle, F.; Grimme, S. Automated Exploration of the Low-Energy Chemical Space with Fast Quantum Chemical Methods. Phys. Chem. Chem. Phys. 2020, 22 (14), 7169-7192.

(38) Groom, C. R.; Bruno, I. J.; Lightfoot, M. P.; Ward, S. C. The Cambridge Structural Database. Acta Crystallogr. Sect. B Struct. Sci. Cryst. Eng. Mater. 2016, 72 (2), 171-179.

(39) Virtanen, P.; Gommers, R.; Oliphant, T. E.; Haberland, M.; Reddy, T.; Cournapeau, D.; Burovski, E.; Peterson, P.; Weckesser, W.; Bright, J.; van der Walt, S. J.; Brett, M.; Wilson, J.; Millman, K. J.; Mayorov, N.; Nelson, A. R. J.; Jones, E.; Kern, R.; Larson, E.; Carey, C. J.; Polat, I.; Feng, Y.; Moore, E. W.; VanderPlas, J.; Laxalde, D.; Perktold, J.; Cimrman, R.; Henriksen, I.; Quintero, E. A.; Harris, C. R.; Archibald, A. M.; Ribeiro, A. H.; Pedregosa, F.; van Mulbregt, P. SciPy 1.0: Fundamental Algorithms for Scientific Computing in Python. Nat. Methods 2020, 17 (3), 261-272.

(40) Havel, T. F. Distance Geometry: Theory, Algorithms, and Chemical Applications. In Encyclopedia of Computational Chemistry; John Wiley \& Sons, Ltd: Chichester, UK, 2002.

(41) Hagberg, A.; D, S.; Swart, P. Exploring Network Structure, Dynamics, and Function Using NetworkX. In Proceedings of the 7th Python in Science conference; 2008; pp 11-15.

(42) Crabtree, J. D.; Mehta, D. P. Automated Reaction Mapping. J. Exp. Algorithmics 2009, 13, 1.15.

(43) Dijkstra, E. W. A Note on Two Problems in Connexion with Graphs. Numer. Math. 1959, 1 (1), $269-271$.

(44) Goodman, J. M.; Silva, M. A. QRC: A Rapid Method for Connecting Transition Structures to Reactants in the Computational Analysis of Organic Reactivity. Tetrahedron Lett. 2003, 44 (45), 8233-8236.

(45) Deserno, M. How to Generate Equidistributed Points on the Surface of a Sphere. In If Polymerforshung (Ed.); 2004; p 99.

(46) Hamlin, T. A.; Swart, M.; Bickelhaupt, F. M. Nucleophilic Substitution (S N 2): Dependence on Nucleophile, Leaving Group, Central Atom, Substituents, and Solvent. ChemPhysChem 2018, 19 (11), 1315-1330.

(47) Ferro-Costas, D.; Martínez-Núñez, E.; Rodríguez-Otero, J.; Cabaleiro-Lago, E.; Estévez, C. M.; Fernández, B.; Fernández-Ramos, A.; Vázquez, S. A. Influence of Multiple Conformations and Paths on Rate Constants 
and Product Branching Ratios. Thermal Decomposition of 1-Propanol Radicals. J. Phys. Chem. A 2018, 122 (21), 4790-4800.

(48) Lee, C. W.; Taylor, B. L. H.; Petrova, G. P.; Patel, A.; Morokuma, K.; Houk, K. N.; Stoltz, B. M. An Unexpected Ireland-Claisen Rearrangement Cascade During the Synthesis of the Tricyclic Core of Curcusone C: Mechanistic Elucidation by Trial-and-Error and Automatic Artificial Force-Induced Reaction (AFIR) Computations. J. Am. Chem. Soc. 2019, 141 (17), 6995-7004.

(49) Franke, R.; Selent, D.; Börner, A. Applied Hydroformylation. Chem. Rev. 2012, 112 (11), 5675-5732.

(50) Heck, R. F.; Breslow, D. S. The Reaction of Cobalt Hydrotetracarbonyl with Olefins. J. Am. Chem. Soc. 1961, 83 (19), 4023-4027.

(51) Kégl, T. Computational Aspects of Hydroformylation. RSC Adv. 2015, 5 (6), 4304-4327.

(52) Decker, S. A.; Cundari, T. R. DFT Study of the Ethylene Hydroformylation Catalytic Cycle Employing a $\mathrm{HRh}(\mathrm{PH} 3$ ) 2 (CO) Model Catalyst. Organometallics 2001, 20 (13), 2827-2841.

(53) Maeda, S.; Morokuma, K. Toward Predicting Full Catalytic Cycle Using Automatic Reaction Path Search Method: A Case Study on $\mathrm{HCo}(\mathrm{CO}) 3$-Catalyzed Hydroformylation. J. Chem. Theory Comput. 2012, 8 (2), 380-385.

(54) Guan, Y.; Ingman, V. M.; Rooks, B. J.; Wheeler, S. E. AARON: An Automated Reaction Optimizer for New Catalysts. J. Chem. Theory Comput. 2018, 14 (10), 5249-5261.

(55) Jones, J. H. The Cativa Process for the Manufacture of Acetic Acid. Platin. Met. Rev. 2000, 44 (94-105).

(56) Armstrong, A.; Boto, R. A.; Dingwall, P.; Contreras-García, J.; Harvey, M. J.; Mason, N. J.; Rzepa, H. S. The Houk-List Transition States for Organocatalytic Mechanisms Revisited. Chem. Sci. 2014, 5 (5), 2057-2071.

(57) Mieusset, J.-L.; Brinker, U. H. The Carbene Reactivity Surface: A Classification. J. Org. Chem. 2008, 73 (4), $1553-1558$.

(58) Domingo, L. R.; Sanz-Cervera, J. F.; Williams, R. M.; Picher, M. T.; Marco, J. A. Biosynthesis of the Brevianamides. An Ab Initio Study of the Biosynthetic Intramolecular Diels-Alder Cycloaddition. J. Org. Chem. 1997, 62 (6), 1662-1667.

(59) Schneebeli, S. T.; Hall, M. L.; Breslow, R.; Friesner, R. Quantitative DFT Modeling of the Enantiomeric Excess for Dioxirane-Catalyzed Epoxidations. J. Am. Chem. Soc. 2009, 131 (11), 3965-3973.

(60) Jahnke, E.; Tykwinski, R. R. The Fritsch-Buttenberg-Wiechell Rearrangement: Modern Applications for an Old Reaction. Chem. Commun. 2010, 46 (19), 3235.

(61) Neese, F. An Improvement of the Resolution of the Identity Approximation for the Formation of the Coulomb Matrix. J. Comput. Chem. 2003, 24 (14), 1740-1747.

(62) Neese, F.; Wennmohs, F.; Hansen, A.; Becker, U. Efficient, Approximate and Parallel Hartree-Fock and Hybrid DFT Calculations. A 'Chain-of-Spheres' Algorithm for the Hartree-Fock Exchange. Chem. Phys. 2009, 356 (1-3), 98-109.

(63) Perdew, J. P.; Burke, K.; Ernzerhof, M. Generalized Gradient Approximation Made Simple. Phys. Rev. Lett. 1996, 77 (18), 3865-3868.

(64) Adamo, C.; Barone, V. Toward Reliable Density Functional Methods without Adjustable Parameters: The PBE0 Model. J. Chem. Phys. 1999, 110 (13), 6158-6170.

(65) Grimme, S.; Antony, J.; Ehrlich, S.; Krieg, H. A Consistent and Accurate Ab Initio Parametrization of Density Functional Dispersion Correction (DFT-D) for the 94 Elements H-Pu. J. Chem. Phys. 2010, 132 (15), 154104.

(66) Grimme, S.; Ehrlich, S.; Goerigk, L. Effect of the Damping Function in Dispersion Corrected Density Functional Theory. J. Comput. Chem. 2011, 32 (7), 1456-1465.

(67) Weigend, F.; Ahlrichs, R. Balanced Basis Sets of Split Valence, Triple Zeta Valence and Quadruple Zeta Valence Quality for H to Rn: Design and Assessment of Accuracy. Phys. Chem. Chem. Phys. 2005, 7 (18), 3297.

(68) Andrae, D.; Häußermann, U.; Dolg, M.; Stoll, H.; Preuß, H. Energy-Adjusted Ab Initio Pseudopotentials for the Second and Third Row Transition Elements. Theor. Chim. Acta 1990, 77 (2), 123-141.

(69) Weigend, F. Accurate Coulomb-Fitting Basis Sets for H to Rn. Phys. Chem. Chem. Phys. 2006, 8 (9), $1057-$ 1065 . 


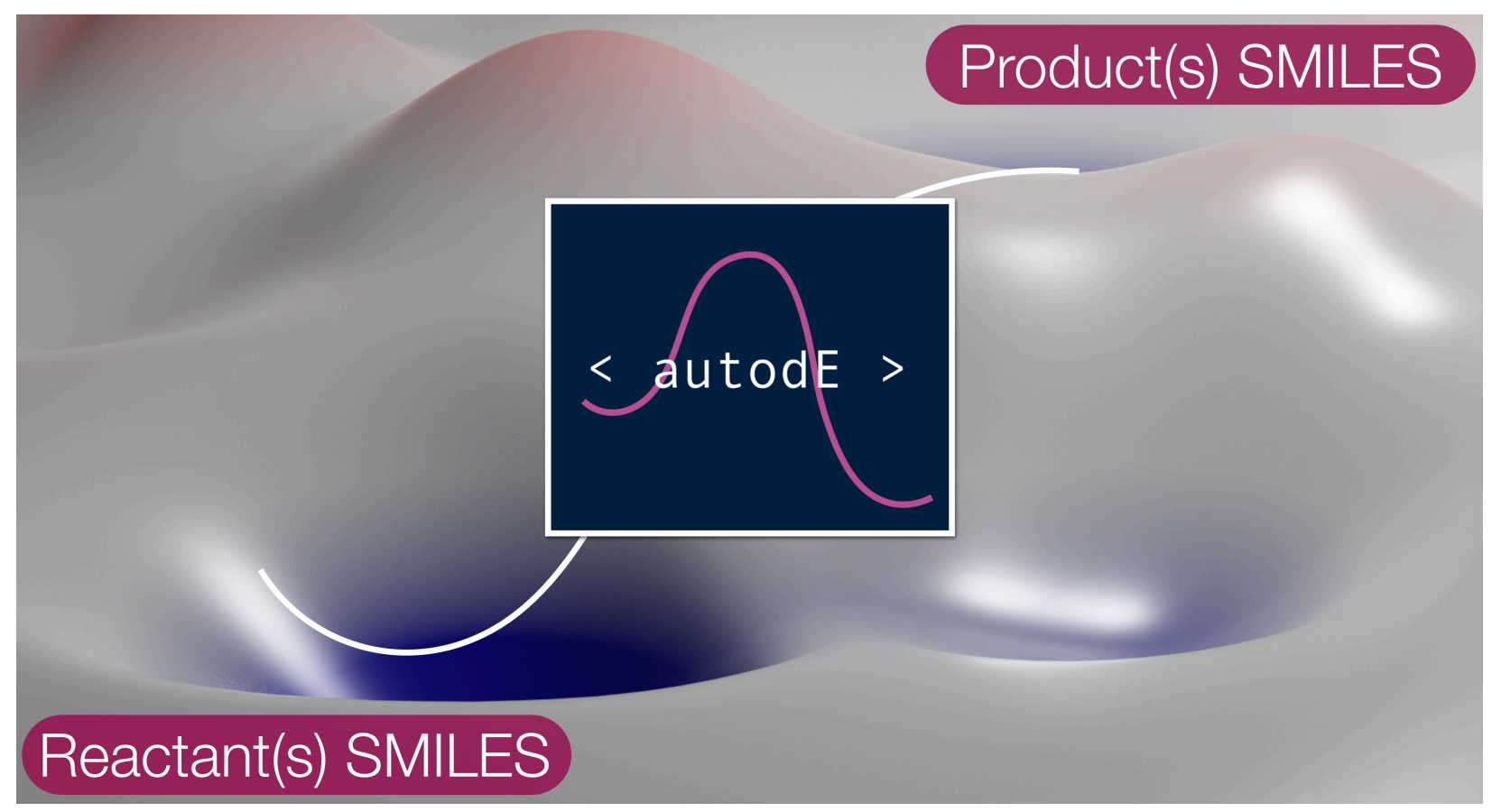

